\title{
"Yo me defiendo": entendiendo la informalidad laboral a partir del trabajo de las mujeres mototaxistas en Barranquilla, Colombia*
}

\author{
DOI: https://doi.org/10.18046/recs.iEspecial.3223
}
Understanding Labor Informality Based on the Work of Moto-Taxi done by Women in Barranquilla (Colombia)

\author{
Lina Buchely ${ }^{* *}$ \\ Universidad Icesi (Cali, Colombia)
}

María Victoria Castro ${ }^{* * *}$

Universidad de los Andes (Bogotá, Colombia)

\footnotetext{
${ }^{*}$ El trabajo de campo que sustenta estas reflexiones fue desarrollado durante los años 2016 y 2017 como parte del proyecto de investigación de las autoras "Ciudad de las mujeres", proyecto financiado por la Universidad del Norte (Barranquilla) y la Universidad Icesi (Cali). Artículo de investigación recibido el 31.10.2018 y aceptado el 25.04.2019.

** Politóloga y abogada con Maestría en Derecho de la Universidad de los Andes (Colombia). LLM de la Universidad de Wisconsin-Madison (Estados Unidos). Doctora en Derecho por la Universidad de los Andes (Colombia). Profesora asistente y coordinadora del Grupo de Estudios de Género de la Universidad Icesi (Colombia). Correo electrónico: lfbuchely@icesi.edu.co ORCID: https://orcid.org/oooo-0oo2-0500-3820

*** Abogada de la Universidad de los Andes (Colombia). Doctora en Derecho por la misma universidad. Correo electrónico:mv.castro226@uniandes.edu.co ORCID: https://orcid.org/oooo-ooo2-2309-4922
} 


\section{Cómo citar/How to cite}

Buchely, Lina; Castro, María Victoria (2019). "Yo me defiendo": entendiendo la informalidad laboral a partir del trabajo de las mujeres mototaxistas en Barranquilla, Colombia. Revista CS, núm. especial, 23-47. https://doi.org/10.18046/recs.iEspecial.3223 
Este artículo explora la interacción entre la informalidad laboral, las condiciones precarias del trabajo y el género, a través del lente de la experiencia subjetiva de las mujeres mototaxistas en Barranquilla, Colombia. A partir de la información obtenida de entrevistas en profundidad, observación etnográfica y grupos focales, el artículo pone en cuestión las aproximaciones usuales sobre ciudad, género e informalidad laboral. Lo anterior, en la medida en que no representan adecuadamente las experiencias subjetivas de las trabajadoras, aplanan el análisis sobre las consecuencias diferenciales para las mujeres en este tipo de trabajos y producen imágenes de inseguridad e inestabilidad no necesariamente percibidas por ellas. El artículo propone cambiar la mirada de los análisis de género y ciudad, en general, a través de una perspectiva itinerante de los análisis de movilidad. Se exploran aquí tres movimientos: 1) el del trabajo en las ciudades fuera de sus márgenes; 2) la movilidad de las identidades; y 3) la paradoja del cuidado como movimiento circular.

PALABRAS CLAVE:

género, movilidad, ciudad, trabajo informal, precariedad

Through the lens of the subjective experience of women who drive moto-taxis in Barranquilla, Colombia, this article explores the interaction between labor informality, precarious working conditions, and gender. Based on the information obtained from in-depth interviews, ethnographic observation, and focus groups, the article questions the usual approaches to city, gender, and labor informality to the extent that they do not represent properly the subjective experiences of the workers, they flatten the analysis of the differential consequences for women in this type of work, and they produce images of insecurity and instability that are not necessarily perceived by these women. The article proposes a change of perspective in terms of analyses of gender and cities in general through a mobile perspective of the mobility analyses. We explore three movements on mobility: (i) the movement of work in cities outside its margins, (ii) the mobility of identities, and (iii) the paradox of care as a circular movement.

\section{KEYWORDS:}

Gender, Mobility, City, Informal Work, Precariousness 



\section{Introducción}

Los estudios de ciudad, al igual que las ciudades, han tenido un estrepitoso crecimiento. Dentro de ese movimiento, interesan las perspectivas más cualitativas que han migrado de los estudios fijos del espacio (paisajes, lugares, sistemas), las instituciones y los ciudadanos (públicos, derechos y ciudadanía) hacia comprensiones en las que el espacio es un conjunto de ensambles. En esta mirada, los actores, sus interacciones y su trabajo coproducen la ciudad, y las movilidades urbanas son fundamentales porque son las mediadoras activas de esa coproducción que nunca está terminada y siempre se está haciendo.

Las movilidades urbanas median, así, como armazones complejas de actores y actantes que se refieren más a las dinámicas y relaciones entre elementos urbanos en constante producción que a los elementos fijos (Blomley, 2008; Salazar, 2013). Como ensamblajes, las ciudades dan cuenta de recorridos de personas distintas, señales, reglas, prohibiciones, tecnologías, viviendas, que nos permiten "estar".

En este registro, interesa entender las tensiones entre movilidades, dispositivos de gobierno y discursos, para controlar o regular el acceso al trabajo de las mujeres en el medio urbano de Barranquilla. Las viñetas que se presentan en este artículo sirven para mostrar las prácticas cotidianas de la movilidad en la calle, centrada en las subjetividades de las informantes y en las materialidades urbanas, así como en los conflictos y tensiones por el uso y significado de las vías, la movilidad, los medios de transporte y las oportunidades laborales.

En vista de que la movilidad es un fenómeno que puede analizarse desde múltiples miradas (Andreasen; Moller-Jensen, 2017), se eligió aquí la mirada relacional en la que el espacio no es estático ni solamente instrumentalizado, de manera que se apuntó a comprender las maneras en las que se ensamblan los espacios físicos de la ciudad, las prácticas de las personas en la vida cotidiana y las relaciones entre las distintas formas de movilidad en el espacio urbano -física y social-. Específicamente, en este trabajo importan las movilidades urbanas, su rol en los procesos de coproducción del espacio y las subjetividades urbanas, así como su papel en el acceso a trabajo adecuado.

Las movilidades son una entrada interesante para entender los procesos de coproducción del espacio, la identidad y la distribución en ciudades como Barranquilla, orientadas a la expansión y el crecimiento, y que están viviendo una rápida urbanización, precisamente, porque este tipo de ciudades fragmenta la vida urbana, al separar los lugares de vivienda de los de trabajo, descanso y consumo, "[estas ciudades] dividen las familias que viven en sitios distantes, que vuelven a las personas dependientes del tiempo de ocio para poder estar en contacto con los familiares; in- 
volucran a la gente en congestiones y trancones; y encapsulan a la gente en ambientes de movilidad privatizados" (Urry, 2012:59). Asimismo, indagar sobre las movilidades urbanas, su papel en los procesos de coproducción del espacio, las subjetividades urbanas y su papel en el acceso al trabajo adecuado, trae consigo la necesidad de trabajar con sujetos específicos, claramente situados, con características propias.

Lo anterior, precisamente, porque interesa alejarse de la mirada hegemónica dentro de los estudios de ciudad y políticas de transporte que se basan en sujetos universales, abstractos y neutros. Esta mirada de los individuos neutros es útil para producir sujetos y cuerpos marginalizados e inmóviles en el discurso (Adey, 2009), por lo que se defiende siempre la necesidad de aterrizar la investigación y los hallazgos a sujetos sexuados, con atributos sociales específicos, actuando en contextos concretos, con agencia y posibilidades de acción más allá que la de ser simples cuerpos con posibilidad de ser transportados (Buchely, 2012; Buchely; Castro, 2013; Castro; Buchely, 2016; 2018).

Los análisis sobre la ciudad se han "movido", entonces, de la mirada fija a la ciudad como realidad terminada, a la mirada en movimiento. Dentro de ese registro, la movilidad se ha convertido en la buzzword del análisis urbano (Salter, 2013). La ciudad, hace por lo menos cincuenta años, es algo que se mueve. Esta metáfora es también útil para mostrar desplazamientos de ideas, marcos de análisis, juegos de conceptos. En este artículo, se proponen tres "movimientos" sobre los análisis de movilidad en las ciudades.

El primero de ellos es un movimiento sobre el trabajo. Si la ciudad es un ensamble de espacios, necesidades, aspiraciones humanas y necesidades tecnológicas, dos de sus ejes (el espacio y el trabajo) se combinan para producir escenarios poslaborales, en donde las personas trabajan con el movimiento (Merino, 2017). En ciudades con más espacio que líneas de transporte oficial, y más necesidades que trabajos, las mujeres mototaxistas en Barranquilla -el lugar social que se analiza-, desafían los roles tradicionales del género para ejercer esta labor. En ese tránsito, el trabajo se mueve de sus lugares y supuestos tradicionales a lugares inexplorados, que superan las dinámicas empleador-empleado.

Pero la apuesta del movimiento de las mujeres lleva al segundo desplazamiento que se quiere analizar: la movilidad de las identidades. Las mujeres de sectores populares, en ciudades drásticamente adaptadas para el desarrollo y el progreso, mueven sus roles de género para construir escenarios de provisión, trabajo y cuidado sostenibles para ellas (Stryker; Wittle, 2006). Este artículo se concentrará también, entonces, en cómo las mujeres transitan, no sin fricciones, de los lugares de domesticidad y subordinación que les depara la inercia social -en ciudades tensamente estratificadas como las colombianas-, a lugares que ellas narran como "de mayor 
libertad". Es un movimiento del cuidado al mercado. Es un tránsito de identidad de la mujer buena a la mujer mototaxista ("machorra, lesbiana y marimacha", como las llaman sus vecinos).

Sin embargo, la metáfora de la movilidad también permite observar cosas que permanecen inmóviles. Dentro de sus experiencias de trabajo, las mujeres narran historias que las regresan a los escenarios circulares. Después de desafiar el destino del trabajo doméstico, su ventaja como mototaxistas mujeres cae en lo que se llamará "la paradoja del cuidado": tienen más clientes porque son mujeres, más delicadas, más dóciles, más cuidadosas. Ellas mismas reproducen el estereotipo del "cuidado" y reconocen cómo les da más oportunidades, pese a que son conscientes, también, de que no pueden escapar a él. Estos trayectos circulares de lo social muestran que existe una suerte de gravedad en el trabajo de las mujeres, que reta sus propios movimientos: los roles de género son difíciles de mover.

En este proyecto, se usaron varias técnicas etnográficas para observar la realidad de las mujeres mototaxistas en Barranquilla. El mototaxismo es un fenómeno relativamente nuevo en las ciudades de países en vías de desarrollo (como los trayectos conceptuales permiten rastrearlo), regularmente informal, que consiste en el desplazamiento en moto de pasajeros(as) a lugares precisos (Burgos, 2016). En Colombia, el mecanismo es utilizado en la mayoría de ciudades del país, sobre todo en los estratos económicos 1 y 2, que tienen menos conexión con las líneas formales de transporte y menos "capital de motilidad". Pese a haber sido caracterizado como un trabajo precario, ya hay varias investigaciones locales que hablan sobre su potencia en la innovación, construcción de redes y apoyos sociales en las bases (en varias ciudades existen sindicatos) y sofisticación de su operación (con existencia de circuitos, control de tarifas y reglas para usuarios/as) (Castro; Buchely, 2016).

El trabajo de campo que sustenta estas reflexiones fue desarrollado durante los años 2016 y 2017, e incluyó diez jornadas de observación de lugares de mototaxistas en Barranquilla (durante la jornada completa), dos grupos focales con mototaxistas (hombres y mujeres), tres grupos focales con mujeres de la ciudad, seleccionadas en las jornadas de observación, y tres entrevistas en profundidad con mototaxistas

1. El capital de motilidad habla de la comodificación del movimiento, material o virtual (movilidad análoga y digital, dado que también nos movemos por las avenidas de la comunicación: internet, telefonía celular, redes sociales). Con esa expresión, se alude al valor que unas personas tienen de moverse con libertad y flexibilidad, sobre otras. Se denomina "capital" porque la movilidad se convierte en un valor que permite maximizar otros factores que generan renta: tiempo, trabajo, ocio. Al mismo tiempo, se vincula a la clase social (dado que son las clases socioeconómicas altas las que pueden movilizarse con más libertad y, así, reproducir otros capitales que ya tienen). En ese sentido, la motilidad ha sido analizada como una variable regresiva, que reproduce las desigualdades sociales (Alcántara, 2010). 
mujeres. Las entrevistas y grupos focales se procesaron manualmente, organizando los hallazgos en dos categorías emergentes: la dignificación del trabajo (analizada como escenarios poslaborales) y las tácticas de resistencia (analizadas como subpolítica o infrapolítica). La convergencia de esas dos categorías permitió plantear la tercera categoría emergente (que señala tensiones antes que continuidades): la paradoja del cuidado.

Este trabajo está dividido en cuatro secciones. La primera se detiene en la presentación del contexto general sobre las mujeres y el acceso al trabajo y al transporte en la ciudad de Barranquilla. La segunda y tercera sección expondrán, mediante el uso de viñetas etnográficas y fragmentos de entrevistas, los hallazgos de la investigación frente a los esquemas poslaborales del trabajo de las mujeres mototaxistas (primera viñeta), y las tácticas de resistencia que ellas despliegan para poder desarrollar su trabajo (segunda viñeta). Finalmente, en la cuarta sección se analiza la "paradoja del cuidado" como inmovilidad en lo móvil, al hablar de las dificultades que experimentan las mujeres en estos escenarios y desarrollar el análisis general del trabajo.

\title{
Las mujeres en Barranquilla: movilidad social y distribución de las oportunidades laborales
}

\begin{abstract}
A finales del siglo XX, la metropolización es reconocida como una condición presente en algunas grandes aglomeraciones latinoamericanas que desbordan los límites jurisdiccionales. Este proceso de metropolización se caracterizó por un modelo de evolución del poblamiento y de diferenciación espacial interna: fuertes desigualdades de ritmo entre espacios centrales y periferias, una desconcentración de población concomitante con el extendimiento espacial, una dinámica demográfica cada vez más centrífuga y un estancamiento, a veces despoblamiento, de las áreas centrales. Estos procesos de urbanización acelerada sin condiciones de absorción de la mano de obra gracias a la incipiente industrialización, entre otros factores críticos, redundaron en la configuración de ciudades caracterizadas por la marginalización, la metropolización de la pobreza, la segregación y la auto segregación creciente. Las metrópolis latinoamericanas progresivamente desarrollaron un modelo expansivo, con fuerte segregación y fragmentación social; con excesiva dependencia del transporte privado frente al público; con dicotomía del tejido económico; dualización del mercado laboral; realimentación del tejido productivo de carácter informal; suburbanización de la producción industrial y una distribución de centros comerciales, de ocio y universitarios por todo el espacio metropolitano (Hurtado; Hernández; Miranda, 2014: 41).
\end{abstract}


La expansión de las áreas urbanas en América Latina, en las últimas décadas, ha originado el crecimiento acelerado de las ciudades, la generación de regiones metropolitanas y la producción de diferentes tipos de problemas, desde el punto de vista de la administración y sostenibilidad de esquemas de funcionalidad, gobernabilidad, servicios, infraestructura y garantía de las condiciones sociales mínimas a todos los habitantes. Estas ciudades dispersas y grandes producen unos espacios fundamentalmente residenciales y funcionalmente incompletos (no tienen acceso a muchas de las infraestructuras y servicios que las zonas viejas de la ciudad sí), que generalmente se ubican en los bordes de estas y que tienen un gran nivel de dependencia frente al resto de la ciudad.

Se supone que la urbanización y el crecimiento de las ciudades, en términos generales, debería permitir mejores índices de desarrollo en ingresos, salud, acceso a educación y mercado laboral, bienes de consumo y bienestar; sin embargo, en el contexto latinoamericano, el grado de pobreza y la ineficiente distribución de los recursos ha producido estas zonas urbanas con déficits de infraestructura, equipamiento, conectividad, gestión gubernamental, entre otros. La movilidad física a través de las ciudades, como medio para acceder a dichos bienes, servicios y oportunidades, se vuelve central en el análisis de la movilidad social y el efectivo acceso a los mismos. Así, la segregación socioeconómica y espacial condiciona, también, el funcionamiento de las ciudades como Barranquilla.

En este contexto, donde muchas zonas residenciales de los bordes de la ciudad no tienen acceso a vías por donde puedan transitar las rutas de transporte público, los recorridos no son funcionales para el tipo de trayectos, y el costo de acceso a la movilidad para transitar la ciudad es muy elevado en comparación con los ingresos, por lo que el acceso al mercado laboral se ve de entrada afectado. Pero, además, en ese contexto de inequitativo acceso al mercado laboral, las mujeres son más vulnerables; más vulnerables al fenómeno de la pobreza, a la invisibilidad del trabajo doméstico y del cuidado no remunerado, así como son más proclives a sufrir las restricciones de tiempo que el trabajo de cuidado impone. La discriminación laboral se refleja, entonces, en menores tasas de participación, mayores tasas de desempleo, menor acceso a empleos de calidad, menores salarios, falta de autonomía económica, entre otras, aumentando los índices de pobreza de las mujeres en Colombia y de los hogares con jefatura femenina.

Así, según el informe de pobreza monetaria y pobreza multidimensional del Departamento Nacional de Planeación (DNP, 2018), tanto a nivel nacional como en el medio urbano y rural en Colombia, la pobreza de las personas que habitan hogares cuya jefatura recae en una mujer es mayor a la pobreza de los hogares con jefatura 
masculina, la brecha es del $6 \%$ en contextos urbanos y, aunque en el período 2010 a 2017 la pobreza en hogares con jefatura femenina se ha reducido en 12 puntos porcentuales, la brecha entre ambos tipos de hogares, en el mismo período, aumentó. Como variables destacadas por el DNP para la comprensión de los resultados, están el hecho de que las mujeres generan menos ingresos que los jefes de hogar hombres por la mayor carga de trabajo doméstico y de cuidado que les impide insertarse en los mercados laborales existentes, la poca oferta de oportunidades laborales para mujeres sin educación en funciones diferentes al trabajo doméstico, y la reducida ayuda en las labores domésticas por parte de la pareja -en los casos en que cuentan con una-, todas ellas produciendo una menor capacidad para generar ingresos monetarios.

Desde el punto de vista del acceso a las oportunidades laborales, la mayor vulnerabilidad de las mujeres pobres es una consecuencia, sobre todo, de la carga de trabajo no remunerado de cuidado que deben asumir:

Es importante resaltar que, para el caso de las mujeres, si estas pertenecen a hogares pobres y son jefes de hogares, dedican en promedio 9 horas más a actividades de cuidado con respecto a mujeres jefes que viven en hogares no pobres. Mientras que si son mujeres cónyuges en hogares pobres dedican en promedio 12,1 horas más con respecto a las mujeres cónyuges de hogares no pobres (DNP, 2018: 62).

De ahí que no sea sorprendente que, en los resultados, para agosto de 2018, de la Gran Encuesta Integrada de Hogares (GEIH) del Departamento Administrativo Nacional de Estadística (DANE, 2018a), que tiene como objetivo principal proporcionar información básica sobre el tamaño y la estructura de la fuerza de trabajo del país, el $55 \%$ de la población desocupada y el $66 \%$ de la económicamente inactiva sean mujeres. La brecha en la participación en el mercado laboral, en Colombia, según la misma encuesta, es del $22 \%$, y la cifra de desempleo es del $12 \%$, para mujeres, frente al $7 \%$, para hombres. El desempeño del Atlántico, en el contexto nacional, no es malo: frente a un promedio nacional de la tasa de desempleo, para 2017, del 9,4\%, este departamento se ubicó por debajo, con una tasa del 7,4 \% (DANE, 2018a); sin embargo, de esa participación en el mercado laboral, solo el $43 \%$ de los trabajadores son mujeres (a pesar de que representan el $51 \%$ de la población del departamento), y el 55,5\% de la población ocupada en Barranquilla se encuentra en esquemas informales (DANE, 2018b).

En ese contexto, donde Barranquilla reduce sus tasas de desempleo, manteniéndose como la ciudad con menor desempleo en el país, al tiempo que aumenta las tasas de informalidad a niveles alarmantes, actividades como el mototaxismo prosperan: su aumento se asocia a la posibilidad de consecución de motos de bajo costo 
que pueden emplearse fácilmente como herramienta de trabajo. Dicha actividad, aunque ha sido catalogada por el Gobierno nacional como un problema social que debe erradicarse a través de normas que la prohíban, permite a quienes la ejercen acceder a ingresos de entre 40.000 y 80.000 pesos diarios (dependiendo del número de horas que se trabaje al día); así como ha generado, en las ciudades donde hay presencia, toda una economía de la cual hacen parte usuarios, conductores, propietarios, talleres, distribuidores y ensambladoras, entre otros actores.

En Barranquilla, la actividad del mototaxismo es reconocida como masculina, de manera que también en ella las mujeres ocupan una posición subalterna y sufren con mayor intensidad la precariedad, el desempleo y la informalidad. En 2012, la fundación Despacio llevó a cabo una caracterización de los mototaxistas en varias ciudades latinoamericanas y, en el caso de Barranquilla, encontraron que el 100 \% de los mototaxistas eran hombres (Rodríguez; Santana; Pardo, 2015).

Sin embargo, el mototaxismo tiene unas ventajas que, desde el punto de vista de las barreras de acceso al mercado laboral de las mujeres, la hacen una actividad interesante. En primer lugar, a diferencia del trabajo doméstico remunerado en las casas de otras mujeres, el mototaxismo como oficio permite flexibilidad en los horarios (pudiendo las mujeres negociar sus cargas desproporcionadas en el trabajo de cuidado, sobre todo las jefas de hogar); en segundo lugar, también a diferencia del trabajo doméstico, el oficio del mototaxismo le da un sentido de dignidad al trabajo de las mujeres, que resuelve la sensación de maltrato y discriminación que la mayoría de trabajadoras domésticas debe soportar.

Una manera de pensar en las características de estas dos opciones laborales para las mujeres barranquilleras es, precisamente, a la luz de la idea de los marcos estructurales que precarizan el trabajo, entre otras razones porque producen riesgos laborales que deben ser soportados por las mujeres. Se sigue aquí el trabajo de Leah F. Vosko (2000), quien utiliza el lente del género para entender las características estructurales del mercado laboral, con el objetivo de identificar sus efectos diferenciales en las mujeres. Vosko (2000) sugiere considerar tres marcos para analizar las condiciones estructurales del trabajo precario: el modelo normativo de empleo, el contrato sexual y las fronteras de la ciudadanía.

El modelo normativo se refiere a la relación estándar de empleo. En el contexto de Barranquilla, como ya se ha presentado, ese estándar beneficia, principalmente, a los hombres, quienes tienen mejor acceso al trabajo formal, de tiempo completo y horarios de trabajo regulares. El contrato sexual (gender contract) opera en paralelo al modelo normativo, para señalar la desproporcionada distribución del trabajo de cuidado entre hombres y mujeres; lo que ubica más fácil a los hombres en la esfera del trabajo remunerado y relega a las mujeres a la esfera de la reproducción social y el 
trabajo no remunerado (Vosko, 200o). Finalmente, con las fronteras de la ciudadanía, Vosko (2000) se refiere a las exclusiones que enfrentan trabajadores migrantes, en particular. Esta mirada de los múltiples marcos permite resaltar que la precariedad cotidiana funciona diferente en la vida de las mujeres, así como opera diferente en la vida de aquellas que se dedican al mototaxismo y de las que se dedican al trabajo doméstico. Todas experimentan presiones y barreras, pero el tipo de presión, de barreras y de capacidad de agenciarlas varía.

Así, el trabajo doméstico tiene unos riesgos laborales que no son visibles para la política pública de la seguridad social, por ejemplo, sobre todo en el caso de las trabajadoras domésticas internas, el hecho de estar sometidas a la imposición de múltiples tareas paralelas (cocinar, limpiar, cuidar niños y mascotas, etc.) (Floro, 1994; 1995; Hopkins, 2017). También hay riesgos laborales no previstos en el caso de las trabajadoras domésticas que trabajan por días con diferentes empleadores; en este caso, el contrato por días implica tener que hacer las diferentes labores en menos tiempo porque las actividades de aseo pesado se condensan en el día en que la empleada va, teniendo ella un mayor desgaste corporal y menor bienestar.

Las mujeres que se dedican al oficio del mototaxismo tienen que lidiar con la radiación solar, la polución y el riesgo de accidentes de tránsito, así como, en el caso de Barranquilla, con la cultura machista y la constante impugnación a su identidad por parte de sus pares hombres, como se verá en las secciones que siguen. Adicionalmente, en el caso de las mujeres mototaxistas, si bien es cierto que son libres de definir sus horarios, también lo es que ellas solas deben soportar las incapacidades o las reducciones en sus ingresos cuando, por razones de fuerza mayor o necesidad, no pueden llevar a cabo su actividad.

Las dos actividades son, entonces, actividades con riesgos superiores y desproporcionados para las mujeres que las llevan a cabo; sin embargo, el tipo de riesgos varía. El espacio del hogar parece un espacio más peligroso para las mujeres que deben trabajar en él que el espacio de la vía pública, para las mujeres que optan por el mototaxismo como fuente de ingresos. Todas ellas experimentan dolor y consecuencias corporales, todas viven en trabajos inseguros y bajo la presión constante; todas tienen trabajos precarios, pero, definitivamente, las mototaxistas en Barranquilla reportan tener más oportunidades. En las siguientes secciones, se presentan los resultados del trabajo de campo que permitió llegar a esa conclusión. Es importante hacer una advertencia: este artículo no pretende "lavarle la cara" al mototaxismo como oficio ideal para las mujeres, pues las condiciones del trabajo son precarias y tremendamente costosas para aquellas que la ejercen (más costosas que para los hombres que lo hacen).

El objetivo es comprender el papel de las movilidades en la constitución de la experiencia de la vida laboral de las mujeres mototaxistas de Barranquilla, y resaltar 
el hecho de que las políticas de fomento de empleo y regularización de los sistemas de transporte deberían 1) tener en cuenta las realidades regionales; 2) concentrarse en las condiciones laborales de las opciones que se ofrecen a las mujeres y no en la generación de empleos, sin evaluar su calidad y características; y 3) organizar la provisión del trabajo de cuidado.

Por tanto, seguir ofreciendo empleos de ocho horas, permitiendo las condiciones indignas y discriminadoras de las trabajadoras domésticas o gastando el presupuesto en cursos de artesanías para convertir a las beneficiarias, madres cabezas de familia, en "mujeres alegres, libres y empoderadas"2 o en "arte-sanas de su futuro"3 (como hacen las oficinas públicas encargadas del género) no soluciona el problema del trabajo precario. De la misma forma que la informalidad no refleja la amplia heterogeneidad de este trabajo en Colombia, la formulación de la política pública es equívoca respecto al tipo de condiciones y calidad en el empleo que las mujeres que deben hacer trabajo de cuidado necesitan. Los testimonios recogidos en este artículo, dados por mujeres mototaxistas en Barranquilla, dan cuenta de estas necesidades.

\title{
Primer movimiento: la movilidad laboral y la informalidad como dignidad y refugio
}

\begin{abstract}
Pregunta: ¿No te cansas?
Respuesta: No, mami, porque a mí me gusta la moto, si no me gustara no hiciera esto. ¿Usted sabe que estar en una casa de familia, que lo humillen a uno por 250.000 pesos [al mes]? En cambio, en dos días me hago eso, máximo en tres días y me levanto a las 10 de la mañana, me acuesto, descanso, me vuelvo a levantar, veo a los niños (mototaxista, comunicación personal, 10.2017).
\end{abstract}

En Barranquilla, se conversó con cinco mujeres mototaxistas que trabajaban en el mismo barrio. En ese lugar y sobre el trabajo de estas mujeres, se realizaron la observación, el grupo focal con mototaxistas y tres entrevistas semiestructuradas posteriores, donde se precisaron la información y las impresiones de las observaciones y los grupos focales. Las cinco mujeres entrevistadas tenían entre 24 y 32 años, todas ellas vivían en barrios estrato 1 de Barranquilla y Puerto Colombia, y ninguna de ellas era propietaria de la moto que manejaba, sino que la alquilaban a

2. Ver González (2018), Casas de cultura... (2017), 300 mujeres... (2017).

3. Ver Narváez (2017). 
prestamistas que conocieron por amigos o familiares en sus barrios. Los contratos de arriendo de motores son sencillos: pagan un seguro mensual y una tarifa básica por uso, que puede ser diaria, semanal o mensual ${ }^{4}$.

Dentro de los grupos focales y las entrevistas, el mototaxismo se presentó como una opción de mujeres valientes. Es una acción valiente, si se quiere, porque destruye destinos y estereotipos que estas mujeres reconocían como un peso. Para ellas, representaba una huida del trabajo doméstico, que se ha convertido en la regla de trabajo para mujeres de estratos 1 a 3 en ciudades como Barranquilla. Los escenarios de trabajo como empleadas domésticas son presentados como indignos por las voces de mototaxistas. Indigno es el salario, indigna es la labor, indigna es la movilidad. En contraste, las mujeres perciben el movimiento de una moto, la conquista de la calle y el riesgo de las avenidas como un poder.

Las casas de familia son una especie de cárcel. Una especie de cárcel de donde no se sale. Un lugar de cansancio, de calor, de servicio a otros. Es un lugar donde no se existe. En cambio, pese a las manchas en la cara, los riesgos para el cuerpo y lo hostil del medio, la moto representa libertad. Una superación, como ellas mismas lo narran (mototaxistas, comunicación personal, 11.2017).

Dentro de sus relatos, es claro que todas tienen identificados cuáles son los riesgos de "salir del molde", expresión que ellas utilizan para nombrar sus acciones. No solo la calle es peligrosa para trabajar, sino que tienen que resistir las burlas y los matoneos de sus compañeros de oficio por ser mujeres, ser juzgadas como "machorras" por sus vecinos y soportar contextos de trabajo para los que no están acostumbradas (aire libre, sol inclemente, movimiento continuo y los ojos curiosos de transeúntes sorprendidos). También tienen que asumir el riesgo de los días malos, de las temporadas sin trabajo y las enfermedades inesperadas.

Pese a ello, ser mototaxista "paga". Y es evidente -como lo muestra la primera cita de esta sección- que con la actividad del mototaxi estas mujeres pueden ganar hasta cinco veces más dinero que sus vecinas empleadas en el trabajo doméstico. Pero no es solo este valor el que está en juego en la opción. La movilidad encarna ahí poderes que no se ven y que ellas sienten al montarse en una moto. Las mujeres relatan sus sensaciones de poder de esta manera:

Yo no molesto a mi familia, yo gracias a Dios me devengo de mí misma. Yo a mi mamá le doy 100.000 de arriendo, les doy a mis tres hijos y yo me compro mis cosas y la cervecita y todo. Pero a mí me va bien, gracias a Dios (mototaxista, comunicación personal, 10.2014).

4. Información consignada en el diario de campo, entrada 1 (12.2017). 
Todas hablan de estas opciones de trabajo como liberadoras, por la confluencia de varios elementos: no hay alguien que las esté controlando, no existen jerarquías, no existen horarios ni uniformes, no hay instrucciones ni regaños y, además, tienen la flexibilidad suficiente para manejar su tiempo, estar en el momento preciso en el que sus familias las necesitan en casa, llevar al colegio a sus hijos y a las citas médicas a sus madres. La movilidad es, entonces, una dinámica que empodera como acción, como trabajo y como valor.

Esa sensación de poder pasa por el cuerpo, que ahora es móvil, arriesgado y curtido (todas ríen en el grupo focal cuando hablan de las manchas en la cara, la vestimenta masculina para trabajar y el "jopo" aplastado por las largas jornadas en la motocicleta). También tiene que ver con el objeto mismo, con la moto como vehículo y como tenencia, que con su ruido y velocidad puede transmitir la sensación de poder, control y libertad. Pero, más que eso, por la capacidad que sienten ellas, a través de la movilidad, de maximizar bienes conexos: moverse más les da más tiempo para estar con sus hijos, les da la posibilidad de dormir más, les da la posibilidad de atender a su familia con mayor eficiencia. Les da una sensación de agencia potente. Les entrega una sensación de libertad.

En el libro Maternidad, fraternidad e igualdad: las madres como sujeto político en las sociedades poslaborales, la española Patricia Merino (2017) elabora la etiqueta de lo "poslaboral" como un estadio construcción del mundo del trabajo que ha superado las lógicas del empleo formal. El mundo actual ha abandonado, de muchas maneras, los esquemas de las relaciones laborales tradicionales, imaginados para realidades posindustriales del siglo XIX. Contrario a ellas, las mujeres ya no sirven a las máquinas, trabajando estáticas, en fábricas de producción en serie, con esquemas de superioridad claros, con producciones predecibles, lineales y masivas. Después del quiebre del modelo fordista, hoy el trabajo se mueve en las motos de Rappi, con los carros de Uber, o los subalojamientos de Airbnb. Esa es una informalidad, de muchas maneras, no precaria (permite autonomía, control y flexibilidad). Algo similar es lo que narran las experiencias de las mujeres mototaxistas de Barranquilla.

Esa preferencia de la informalidad, por parte de las mujeres, ha sido bien documentada por los análisis de trabajo femenino en el mundo contemporáneo (Porras, 2018). En este mismo número, el trabajo de Natalia Ramírez habla de esa "informalidad elegida" como una opción que las mujeres escogen, cada vez más, basadas en la conciliación que les permite hacer de sus esferas de trabajo productivo y reproductivo. Este es un mundo que, como se dijo anteriormente, se ha movido vertiginosamente hacia el "progreso", en muchos campos, pero no ha tenido tanto éxito moviendo a las mujeres de la jaula del cuidado que hace más de cien años describía Engels. Estas mujeres agrietan esa jaula con su trabajo. 
Pero la informalidad no es un rayo en cielo sereno dentro de los escenarios neoliberales. Esta condición entrega una sensación de control, libertad y autonomía inminentes que olvidan temporalidades futuras. Parte de los hallazgos de este trabajo es que, en esta ciudad, las mujeres mototaxistas tiene espacio-tiempos (Valverde, 2015) excesivamente presentes, joviales, inmediatos. No piensan en contingencias (por supuesto que no tienen seguros laborales para accidentes), no se proyectan en el futuro (no cotizan a pensión en un esquema laboral que ha decidido amarrar la seguridad social al contrato de trabajo) y no reparan tampoco en debilidades (ninguna tiene seguro de salud más allá de la cobertura del Sisbén). Los costos de estas actividades informales, como alternativas de trabajo femenino, se verán dentro de veinte y treinta años, cuando estas mujeres con jornadas de trabajo exponenciales $\mathrm{y}$ trayectos hexagonales no puedan acceder a una pensión, por ejemplo.

Este cálculo de los riesgos se exaspera cuando ellas mismas cuentan los peligros a los que la dinámica de la calle y la esquina las somete. Los hombres mototaxistas las rechazan y juzgan como lesbianas por desarrollar este trabajo. Para defenderse de esto, ellas aplican toda clase de tácticas. Una de las informantes contó:

Una pecueca esos hombres, juyy!, yo un día tuve que traer a un malandro porque había uno que me maltrataba verbalmente... me decía: “Te voy a levantar a pata”, y un día fui a buscar a un amigo mío que es bandido y le dije: "Papi, ven acá, me está pasando esto y esto”, y el man me ofrece: “Déjamelo”, él respondió. Pues un día el man llegó y dijo: "Ven acá, el man que se meta con la gorda va a tener problemas conmigo, porque así como el hombre tiene derecho a comer para sus hijos, ella también tiene derecho a trabajar". Y el man ahora come dulce, me da; toma gaseosa, me da. Uno tiene que ser así, porque es que como uno es mujer... yo tiro trompá [trompadas], porque yo me le cuadro, el problema es que un man asígrandote le pega una patada a uno y lo jode, pero a un man chiquitico yo me le cuadro... porque el papá de mis hijos era chiquitico y yo me le cuadraba, pero un man grandote ahí... entonces ya ellos me dicen y ya, yo digo: "Ah, Dios, pelea por mi”" (mototaxista, comunicación personal, 10.2016).

Pero los riesgos de la calle no son solo físicos. Ya se ha hablado de cómo la calle y los dispositivos de socialización controlan los cuerpos y las sexualidades de las mujeres (Castro; Buchely, 2018). Una evidencia de cómo esto ocurre es el juzgamiento con la etiqueta de "lesbianas, machorras y marimachas" a las mujeres mototaxistas. Dentro de los grupos focales, fue evidente que estas acusaciones eran algunas de las que más perturbaban a las mujeres mototaxistas, ya que se gastó un tiempo largo hablando solo de esta forma de estigmatización y el costo que para ellas tiene operar una suerte de renuncia a la sexualidad. En los mismos grupos focales, una mujer contó, con tristeza: 
Mi hijo me dice: "Ay, mami, busca otro trabajo, mis amiguitos me dicen que tú eres lesbiana”. Él tiene 15 años, el mayor, 17 y la niña, 11. Yo le explico que este es mi trabajo y ya se le ha quitado eso (mira al piso) (mototaxista, comunicación personal, 10.2018).

Este testimonio muestra la fortaleza de los sistemas de control y represión ante las movilidades femeninas, así como la evidencia de las violencias de género amarradas al ejercicio de trabajos no tradicionales para lo femenino y las feminidades contra-hegemónicas que se construyen a través de esta clase de actividades. Pero las mujeres mototaxistas resisten.

\section{Segundo movimiento, el de la identidad: tácticas de resistencia y atajos al control}

Pregunta: Y tú, ¿qué haces?

Respuesta: Yo le digo así al cliente: "Papi, ya sabes si me para el tránsito yo digo que es mi marido y te dejas besar”. Y él se ríe. Y así (...) y les digo: “¿Te vas a ir con un hombre? Vente con una mujer que vas más segura, mi vida” (informante 4, comunicación personal, 09.2016).

Las mujeres mototaxistas de Barranquilla hablan de las múltiples violencias que resisten en su trabajo. Varias contaron que, a las amenazas de golpizas, los insultos y estereotipos sobre su orientación sexual se sumaba también una brecha en sus ganancias: a las mujeres les pagaban menos por carrera y les pedían más fiado. Sin embrago, ellas convertían eso en una ventaja: lo narraban como una generación de confianza y fidelidad con su clientela.

En esta sección, se analizará cómo ese "sin embargo" opera en los relatos de las mujeres. Pese a que existe una violencia estructural que parece regresarlas a los lugares predecibles (el trabajo doméstico, su casa, la casa de sus padres), las informantes se atrincheran en las calles. Despliegan varias tácticas que, como lo indicaba De Certeau (2007), les permiten navegar lo cotidiano, romper la vigilancia, alterar-de manera suplementaria- el orden. Todos ellos son comportamientos mínimos que, como el ejercicio de la táctica señala, juegan con los mecanismos de disciplina, los usan, los subvierten.

En el ejercicio de observación en la estación de mototaxis, se encontró que las mototaxistas de Barranquilla desplegaban tres tácticas fundamentales para resistir las experiencias de exclusión: explotaban su imagen como madres, destacaban el trabajo de cuidado y erotizaban sus intercambios mundanos. La primera táctica 
utiliza los roles e imaginarios tradicionales de las mujeres como formas de mercadeo de su servicio. Las mototaxistas se construyen como madres al volante: varias hacen trayectos exclusivos de niños al colegio o son especialistas en mujeres "entaconadas y bonitas, que no se les suben a los manes" (informante 2, comunicación personal, 09.2016). Para afianzar el mensaje, ellas hacen referencia a varias cosas: su cuidado con la velocidad, su cumplimiento de las normas y su trato suave.

A mí todas esas señoras me piden que lleve a los hijos. Inclusive la semana pasada empecé con un niño especial que hay que llevarlo con cuidado (...). Yo esquivo bien piedras y huecos, hasta cuando sola porque se maltratan los huesos (informante 1, comunicación personal, 09.2016).

Las mujeres al volante, entonces, son "buenas madres". Son aquello de lo que, paradójicamente, buscan huir. La domesticidad las alcanza en sus motos.

Por su parte, la segunda táctica habla de la disponibilidad y el trabajo de cuidado. Para construir fidelidad con los clientes y amistad con los compañeros rudos, las mujeres mototaxistas hacen fritos, llevan almuerzos, hacen domicilios y dan su WhatsApp para servicios especiales. Así, dicen, sus clientes valoran de ellas esos comportamientos amigables y empáticos.

Hay una estación que yo paso, me dicen "escobita", tú sabes que escoba barre de todo. Yo los recojo a todos, no importa. A mí me va bien, gracias a Dios. Los sábados y domingo me gano más plata; me hago 160, 180. Porque es que yo me voy lejos, yo me voy pa'l norte y ellos no se van. Y voy sabroso, a donde la gente llame, donde la gente necesite (...).

Hay uno que dice: “Oye, gorda, aquí queremos tener una mujer”. Pero, jay!, yo ya estoy acostumbrada a mi estación (...).

Hay otros amigos que yo les llevo, cuando hago chicharrón les llevo (informante 2, comunicación personal, 09.2016).

Esa disponibilidad constante también es costosa para estas mujeres. Ellas cuentan cómo tienen problemas con sus parejas y familias por su constante disponibilidad frente al trabajo y los denominados "servicios especiales". Sus parejas les reclaman, por ejemplo, no parar de trabajar nunca, estar siempre "pegadas del teléfono" o "haber cambiado la familia por la plata" (grupo focal, comunicación personal, 10.2018). Esto es importante para los hallazgos de la investigación porque señala una especie de continuo entre el mundo del trabajo y la familia, muy visible para las experiencias femeninas y, también, muy sancionado socialmente, en los flujos cotidianos de control. 
La tercera táctica tiene que ver con la erotización de los espacios de trabajo. Las mototaxistas barranquilleras cuentan, entre risas, por ejemplo, que a ellas no les ponen comparendos porque "lo saben manejar" con los policías.

Cuando hay que usar las herramientas, se usan. Uno juega con ellos, ellos juegan con uno (...). Imagine uno jodido, tiene que usar lo que sea. Cuando hay que sacar la pierna, hay que sacarla (informante 1, comunicación personal, 09.2016).

Los diarios de campo de las observaciones están llenos de interacciones erotizadas. Miradas continuas e intensas, chistes sexuales, interacciones cercanas y corporales. Pese a ello, estas mujeres no reportan tener miedo o temor frente al acoso sexual callejero. Todo lo contrario, parecen disfrutar y saber usar, en un balance complejo, la erotización como una herramienta de trabajo.

\section{Tercer movimiento: la “inmovilidad” de la paradoja del cuidado}

La descripción de las tácticas anteriores lleva a plantear lo que se llamará la "paradoja del cuidado". Fue visible, durante el trabajo de campo, que las mujeres resistían desde abajo los estereotipos e inercias sociales que las ponían en roles hegemónicos. Esas resistencias, además, resultaban siempre conspicuas, suplementarias, encubiertas, como son las luchas en lo cotidiano. No había entre ellas un "plan o estrategia claro" para enfrentar a esos hombres rudos en las esquinas. Había comida, cariño y sexo, y eso era lo que se usaba.

El carácter mundano de esas resistencias, sin embargo, llevó a cuestionar su contenido simbólico. Era paradójico que, queriendo huir de la domesticidad, estas mujeres acabaran atrapadas en los estereotipos, ahora de manera consciente: ser madres, ser cuidadoras, ser honradas, ser bondadosas, ser incondicionales. En sus relatos, se reconocía la fuerza de una estructura difícil de minar, que muestra una robusta matriz con roles precisos de género, en los que las mujeres solo pueden ser eso: madres y amantes. En ese sentido, la movilidad les jugaba a estas mujeres una mala pasada. No podían moverse de la identidad hegemónica porque, de alguna manera, las regresaban a ella, con fuerza. La identidad, en este sentido, opera como una gravedad.

Esa gravedad, sin embrago, es relacional. La paradoja del cuidado refuerza una identidad maternal y de cuidado frente a sus colegas hombres del mototaxismo, dado que ellos establecen normas informales que excluyen a las mujeres. Las resistencias 
son contradictorias en el sentido que refuerzan un patrón de mirada patriarcal, como efecto reflejo a un espacio de libertad que ellas mismas han creado. Es un efecto reactivo.

La negociación con el patriarcado se da, entonces, entre complejas cesiones y concesiones frente a la estructura, desde las grietas que evidencian su debilidad. En ese sentido, las identidades tradicionales también son usadas como instrumentos para negociar espacios de subversión. Estos son procesos contradictorios que refuerzan estereotipos, pero, a su vez, juegan con ellos, mostrando un tipo de agencia en entornos muy difíciles (Arango; Pineda, 2012). Las categorías de sub e infrapolítica ayudarán a entender este hallazgo en la sección siguiente.

\section{Precisiones finales: la ciudad de las mujeres y el movimiento}

Este trabajo empezó usando la metáfora del movimiento para analizar las experiencias de las mujeres que trabajan en el mototaxismo en Barranquilla. La primera de ellas habló de un movimiento sobre el trabajo. La exploración del campo que se presenta mostró cómo las mujeres mototaxistas, en esta ciudad, desafían los roles tradicionales del género y el trabajo, estableciendo dinámicas complejas que desdibujan los espacios laborales de desempeño tradicionales, las divisiones entre los ejercicios laborales y no laborales, y las lógicas de dependencia, jerarquía y subordinación. Usando las motos como fuente de poder y las ciudades como escenario, las mototaxistas aceleran y aguantan el sol para poder estar más tiempo con su familia, garantizarse más tiempo de descanso, más autonomía y una sensación mayor de liberad y control sobre sus vidas, sus tiempos y sus cuerpos.

El movimiento, en relación con el trabajo informal, habla de esos desplazamientos desde el mundo laboral formal hacia nuevos escenarios de ejercicios del trabajo que, de la mano de las lógicas femeninas, desestructuran las premisas claves de la formalidad: diferencia entre espacio de trabajo y espacio privado, diferencia entre tiempo de trabajo y tiempo privado, jerarquía, control y cesión de libertad al empleador.

El segundo desplazamiento habla de la movilidad de las identidades. El traslado hacia la autonomía del trabajo en las motos señala otros movimientos visibles para las mujeres barranquilleras: aquel que va de la pasividad a la acción, de la heterosexualidad obligatoria a la identidad de "machorras". Este texto habla también de cómo las tácticas de trabajo que despliegan las mujeres mototaxistas sufren una inmediata sanción por parte de la estructura heteronormativa: son agredidas, tildadas de "lesbianas, machorras y marimachas", en una reacción que busca la retracción de su movilidad, su parálisis en el rol de mujeres tradicionales. 
El tercer movimiento habla de la circularidad o de la inmovilidad: de la inmovilidad del cuidado. De la mano de las dos movilidades anteriores, el movimiento circular hace referencia a la paradoja de la inmovilidad del trabajo de cuidado. Las mujeres se ven perseguidas por el rol de madre, las experiencias de cuidadoras y la habilidad por las actividades de la reproducción. En este sentido, el plus en el mercado que tienen las mujeres mototaxistas en Barranquilla, el beneficio principal de los movimientos anteriores (de la movida a la informalidad y del juego con la identidad) es que pueden ser buenas madres (lo que fija, antes que desestabilizar, el análisis binario): cuidar a los niños, no violar a las mujeres, manejar con el cuidado de "una mujer".

La ciudad y el movimiento de las mujeres permiten, entonces, hablar de cómo ellas, con sus movimientos (en forma de resistencias, tácticas y desafíos), retan los espacios y supuestos del derecho laboral. En ese contexto y de la mano con esos movimientos, las experiencias con las mujeres mototaxistas de Barranquilla permitieron llegar a cuatro conclusiones sobre su vida y las maneras en que negocian los ingresos, el trabajo y sus responsabilidades.

La primera tiene que ver con las experiencias espaciales circulares. Los trayectos de las mujeres, que conectan el trabajo y el cuidado, no son trayectos lineales, directos y siempre crecientes. Por fuera de las dinámicas y métricas de los sistemas de transporte, las mujeres ensamblan movilidad y espacios de forma sinuosa y curva. Sus rutinas hablan de trayectos de trabajo interrumpidos por visitas a sus casas, por las rutinas de sus hijos y de sus padres, y por las dinámicas de cuidado. En ese sentido, una mototaxista dijo, dentro de uno de los grupos focales, que ella era "ratera", haciendo referencia a que trabaja por ratos, interrumpiendo siempre la jornada para darle tiempo a su familia. Esa expresión condensa a lo que se hace referencia: su trabajo tiene una temporalidad inmediata con una espacialidad siempre convergente. Todos sus trayectos conducen a su casa, varias veces al día, donde la moto y el celular (ambos actantes de la velocidad) les permiten estar de manera casi simultánea. No hay una ciudad lineal y temporalmente fragmentada para las mujeres.

La agencia frente a este trabajo flexible es otro hallazgo. El derecho laboral languidece con esquemas que, como los femeninos, son carentes de jornadas precisas, períodos, reportes verticales e instrucciones jerárquicas; frente a estos escenarios, las mujeres construyen estilos de trabajo con feroz autonomía. El trabajador que emerge de las realidades que imaginó el derecho laboral tradicional-del siglo pasado-es, por supuesto, un hombre de clase media, profesionalizado, que tiene unas experiencias de vida particulares. Estas mujeres, por el contrario, han mostrado cómo sus realidades retan esa existencia del trabajo. Contrarias a los modelos tradicionales, sus prácticas de trabajo muestran un espectro denso entre el trabajo productivo y reproductivo, entre la provisión y la familia, entre la intimidad y el mundo de lo 
público. Ese entrecruzamiento, como centro de las lógicas de trabajo, es algo que falta por analizarse, dentro de esquemas femeninos de experiencias laborales que superan las lógicas de comprensión.

Además, las experiencias de las informantes muestran la potencia de los objetos en el ensamblaje de las ciudades. Para esas mujeres, las motos, los celulares y el dinero operan como actantes que hacen algo en sus vidas: les dan el poder de la velocidad, la presencia intensa de las redes y la seguridad de la autonomía económica. Las motos hacen cosas (producen sensaciones de poder, autonomía y libertad, todas adscritas a la motilidad), los celulares construyen una realidad paralela y asequible (en donde se ofrecen servicios especiales y se está siempre disponible, para clientes y familia), y el dinero genera efectos en sus cuerpos y valía (las hace sentir autónomas e independientes de sus familias y parejas). Esta reflexión sobre los actantes es otro eje pendiente por desarrollar en los análisis de las ciudades, en los que humanos se oponen a espacios, en dicotomías absolutas que no permiten observar que los objetos "hacen" cosas.

Finalmente, las mujeres mototaxistas de Barranquilla cuentan una historia poderosa en torno a la infrapolítica (Scott, 200o). Con tácticas y atajos, ellas logran conspirar con una realidad que, por gravedad, las ubica en lugares incómodos: las casas de familia, los esquemas domésticos. Pese a ello, establecen negociaciones interesantes con el patriarcado, donde ceden y reproducen roles tradicionales, a cambio de sus propias sensaciones de control y libertad. Ser mototaxista es una identidad móvil, que negocia la imagen de la mujer machorra (masculina) con la mujer tradicional (cuidadora y honrada). Estos son modelos de feminidades no hegemónicas que establecen una política encubierta, un disfraz. Esas no son mujeres luchando por sus derechos, participando en la política institucional. Son mujeres que subvierten sus identidades desde sus cotidianos, con luchas suplementarias, discretas (Laclau, 2001), desde una experiencia micro e informal que siempre oculta su poder subversivo, al mismo tiempo que genera las condiciones para él.

Estas mujeres mototaxistas son, entonces, un contrapoder. El contrapoder es entendido aquí como una suerte de conspiración subterránea de los subalternos frente a la dominación, que, tras resultar hegemónica, hace muy costosa la resistencia "pública y directa". Las mujeres resisten "haciéndose las tontas" y colaborando con el patriarcado. Las elaboraciones teóricas de la subpolítica, la infrapolítica o los contrapoderes (Scott, 2000) suponen que todos entienden la dominación y le siguen el juego, porque es inescapable. Pese a ello, pese al reconocimiento como dependientes y subordinados, se esconden las prácticas y tácticas de resistencia (Falleti, 2008). Esto implica que la dominación, entonces, nunca es tan sólida como parece y siempre resulta incompleta. 
Por ello, los atajos de las mujeres mototaxistas de Barranquilla se hacen posibles y logran ensamblarse en experiencias de ciudades intensas, como las que se han descrito. Los movimientos analizados como hallazgos de este trabajo así lo cuentan: la movilidad de las mujeres hacia un registro que escapa el derecho laboral (donde lo formal y lo informal se colapsan), la movilidad hacia identidades contrahegemónicas de la feminidad (que ratifican y, al mismo tiempo, retan los roles tradicionales) y la inmovilidad del cuidado, que muestra cómo, pese a las resistencia y a las huidas, el trabajo de cuidado es una realidad que todavía determina el cotidiano de las mujeres.

\section{Referencias}

300 mujeres del programa "De la mano con la Primera", capacitadas en artesanías en Casas de Cultura (23 de octubre de 2017). Zona Cero. Recuperado de http://zonacero.com/generales/30o-mujeres-del-programa-de-la-mano-con-la-primera-capacitadas-en-artesanias-en-casas-de

Adey, Peter (2009). Mobility. Abingdon: Routledge.

Alcantara de Vasconcellos, Eduardo (2010). Análisis de la movilidad urbana. Espacio, medio ambientey equidad.CAF. Recuperado de http://www.scioteca.caf.com/handle/123456789/414

Andreasen, Manja Hoppe; Moller-Jensen, Lasse (2017). Access to the City: Mobility Patterns, Transport and Accessibility in Peripheral Settlements of Dar es Salaam. Journal of Transport Geography, 62, 20-29. https://doi.org/10.1016/j.jtrangeo.2017.05.005

Arango-Gaviria, Luz Gabriela; Pineda D., Javier A. (2012). Género, trabajo y desigualdades sociales en peluquerías y salones de belleza de Bogotá. Revista CS, 10, 93-130. https://doi. org/10.18046/recs.110.1356

Blomley, Nicholas (2008). Simplification is Complicated: Property, Nature, and the Rivers of Law. Environment and Planning A, 4O(8), 1825-1842. https://doi.org/10.1068/a40157

Buchely, Lina (2012). El precio de la desigualdad. Análisis de la regulación del trabajo doméstico desde el DDL. Estudios Socio-Jurídicos, 14(2), 107-143.

Buchely, Lina; Castro, María Victoria (2013). La reforma pensional y las mujeres: ¿Cuándo será que dejarán de 'hacernos el favor'? Precedente, 2, 205-226.

Burgos, Sonia (2016). El mototaxismo: un medio de transporte urbano como alternativa económica en San Juan de Pasto. Revista de Sociología, Departamento de Sociología, Universidad de Nariño, $V$, 11-27. 
Casas de cultura de Barranquilla capacitan en artesanías a 300 mujeres (23 de octubre de 2017). ExtraNoticias. Recuperado de http://extranoticias.com.co/sitio/casas-cultura-barranquilla-capacitan-artesanias-30o-mujeres

Castro, María Victoria; Buchely, Lina (2016). Mujer, espacio y poder: ciudad y transporte público como dispositivos de exclusión. Reflexiones desde la ciudad de Cali. Estudio de caso de los "motorratones", carros piratas y usuarias del servicio en las comunas 15 y 18 de la ciudad de Cali. Estudios Socio-Jurídicos, 18(2), 229-254. https://doi.org/10.12804/esj18.02.2016.08

Castro, María Victoria; Buchely, Lina (2018). Cities and the City: Spatiotemporal Imaginaries of Class and Gender in Barranquilla and Cali, Colombia. Gender, Place \& Culture, 25(12), 1719-1737. https://doi.org/10.1080/0966369X.2018.1554556

De Certeau, Michael (2007). La invención de lo cotidiano. México D.F: Universidad Iberoamericana.

Departamento Administrativo Nacional de Estadística (2018a). Mercado Laboral por departamentos. Año 2017. Recuperado de https://www.dane.gov.co/index.php/estadisticas-por-te$\mathrm{ma} / \mathrm{mercado}$-laboral/mercado-laboral-por-departamentos

Departamento Administrativo Nacional de Estadística (2018b). Medición de empleo informaly seguridad social. Trimestre móviljunio-agosto 2018. Recuperado de https://www.dane.gov.co/files/ investigaciones/boletines/ech/ech_informalidad/bol_ech_informalidad_jun18_ago18.pdf

Departamento Nacional de Planeación (2018). Pobreza monetaria y pobreza multidimensional. Análisis 201O-2017. Recuperado de https://colaboracion.dnp.gov.co/CDT/Desarrollo\%2O Social/Pobreza\%2oMonetaria\%20y\%2oMultidimensional\%2oen\%2oColombia\%20 2010-2017.pdf

Falleti, Valeria (2008). Reflexión teórica sobre el proceso sociopolítico y la subpolítica. Un estudio de caso: el "cacerolazo" y las asambleas barriales. Revista Mexicana de Sociología, 7o(2), 361-398. Recuperado de http://www.scielo.org.mx/scielo.php?script=sci_arttext\&pid=So188-25032008000200005\&lng=es\&tlng=pt

Floro, María Sagrario (1994). Work Intensity and Women's Time Use. En Color, Class and Country: Experiences of Gender (pp. 162-181), editado por Gay Youngand; Bette Dickerson. London: Zed Press.

Floro, María Sagrario (1995). Women's Well-being, Poverty, and Work Intensity. Feminist Economics, 1(3), 1-25.

González, José (8 de febrero de 2018). Mujeres cabeza de hogar capacitadas en artesanías. La República. Recuperado de https://www.larepublica.co/responsabilidad-social/mujeres-cabeza-de-hogar-capacitadas-en-artesanias-2597497 
Hopkins, Carmen Teeple (2017). Work Intensifications, Injuries and Legal Exclusions for Paid Domestic Workers in Montréal, Québec. Gender, Place \& Culture, 24(2), 201-212. https:// doi.org/10.1080/0966369X.2017.1298573

Hurtado-Tarazona, Adriana; Miranda-Ruiz, Leonel; Hernández-Ospina, Mónica (2014). Gestión de grandes proyectos urbanos en espacios metropolizados: los sistemas integrados de transporte masivo en Colombia. Bogotá: Universidad Piloto de Colombia.

Laclau, Ernesto (2005). La razón populista. Buenos Aires: FCE.

Merino, Patricia (2017). Maternidad, igualdad y fraternidad: las madres como sujeto político en las sociedades poslaborales. Madrid: Clave Intelectual.

Narváez-Ortega, Vivian (5 de noviembre de 2017). Mujeres atlanticenses, "arte-sanas" de su futuro. El Heraldo. Recuperado de https://www.elheraldo.co/entretenimiento/mujeres-atlanticenses-arte-sanas-de-su-futuro- 419820

Porras, Laura (2018). "Viviendo del Rebusque": A Study of How Law Affects Street Rebuscadores in Bogotá (Tesis de doctorado). University of Ottawa, Ottawa, Canadá.

Rodríguez, Daniel; Santana, Manuel; Pardo, Carlos Felipe (2015). La motocicleta en América Latina: caracterización de su uso e impactos en la movilidad en cinco ciudades de la región. Recuperado de http://scioteca.caf.com/handle/123456789/754

Salazar-Arenas, Óscar Iván (2013). De liebres, tortugas y otros engendros: movilidades urbanas y experiencias del espacio público en la Bogotá contemporánea. Revista Colombiana de Antropología, 49(2), 15-40. https://doi.org/10.22380/2539472X.274

Salter, Michael (2013). Justice and Revenge in Online Counter-publics: Emerging Responses to Sexual Violence in the Age of Social Media. Crime, Media, Culture, 9(3), 225-242. https:// doi.org/10.1177/1741659013493918

Scott, James (2000). Los dominados y el arte de la resistencia. México: Era.

Stryker, Susan; Wittle, Stephen (Eds.) (2006) The Transgender Studies Reader. New York: Routledge Taylor \& Francis Group.

Urry, John (2012). Sociology beyond Societies: Mobilities for the Twenty-first Century. Abingdon: Routledge.

Valverde, Mariana (2015). Chronotopes of Law: Jurisdiction, Scale and Governance (Hardback). Recuperado de http://www.bookdepository.com/Chronotopes-Law-Mariana-Valverde/9780415715584

Vosko, Leah F. (200o). Temporary Work: The Gendered Rise of a Precarious Employment Relationship, 11. Toronto: University of Toronto Press. 